\title{
A Two-Phase Model for Personnel Selection Based on Multi-Type Fuzzy Information
}

\author{
Chen-Tung Chen ${ }^{1, *}$ and Wei-Zhan Hung ${ }^{2}$ \\ 1 Department of Information Management, National United University, No.1, Lien-Da, Kung-Ching Li, \\ Miaoli 36003, Taiwan \\ 2 FinTech Innovation Center, National Kaohsiung University of Science and Technology, No. 415, \\ Jiangong Rd., Sanmin Dist., Kaohsiung City 807618, Taiwan; weizhanhung@gmail.com \\ * Correspondence: ctchen@nuu.edu.tw
}

Received: 14 September 2020; Accepted: 30 September 2020; Published: 3 October 2020

\begin{abstract}
From the viewpoint of human resource management, personnel selection is one of the more important issues for enterprises in a high-level competitive environment. In general, many influence factors, quantitative and qualitative, affect the decision-making process of personnel selection. For considering qualitative factors, decision-makers cannot always easily judge the suitable degree of each applicant. Under this situation, this research proposes a systematic decision-making method based on computing with linguistic variables. First, unsuitable applicants are filtered by considering the quantitative information of each applicant. At this stage, technique for order of preference by similarity to ideal solution (TOPSIS) and entropy methods are aggregated to eliminate unsuitable applicants in accordance with their closeness coefficient values. Second, experts (or decision-makers) use different types of 2-tuple linguistic variables to express their opinions of suitable candidates with respect to qualitative criteria. At this stage, we consider different preference functions in the preference ranking organization method for enrichment evaluation (PROMETHEE) method to calculate the outranking index of each suitable candidate. Next, we aggregate the closeness coefficient and outranking index of each suitable applicant to determine the ranking order. In order to illustrate the computational processes, an example demonstrates the practicability of the two-phase personnel selection method. The benefit of the proposed method is as follows. (1) It reduces the time for reviewing and evaluating the huge numbers of applicants. (2) It avoids subjective judgment by experts to determine the weights of all criteria. Finally, conclusions and contributions are discussed at the end of this paper.
\end{abstract}

Keywords: personnel selection; quantitative and qualitative factors; 2-tuple linguistic variable; closeness coefficient; outranking index

\section{Introduction}

Human capital is one of the most important core assets for enterprises competing with other companies to survive in a high-level competitive environment. Personnel recruitment affects the quality of work and the operational performance of an enterprise [1]. In order to enhance an enterprise's competitiveness, it needs to select the right persons for the right jobs [2]. On the contrary, this will reduce the competitiveness of the enterprise and cost a lot of time and money for training workers to operate their jobs well if employees are assigned to the wrong position. Therefore, personnel selection is one of the important decision-making problems for managers of every enterprise. However, many quantitative and qualitative factors should be considered in dealing with the personnel selection problem. By considering the influential factors, a personnel evaluation and selection process can be formed like a multiple-criteria decision-making (MCDM) problem. 
The multi-criteria decision-making (MCDM) is the systematic procedure to find a suitable alternative from a set of feasible alternatives in accordance with multiple evaluation criteria [3,4]. Up to now, MCDM methodologies have been widely used to solve problems in the sciences, business, government, and engineering fields [5] (relative studies in the literature are in Section 2). In fact, some situations arise in the personnel selection problem. First, many applicants compete with each other for some kinds of jobs that have a high salary. Second, all high-level managers do not have enough time to interview all applicants, and the opportunity cost is too high to interview all applicants for a high-level manager position. Third, not all applicants fit the requirement of the job. Therefore, two-phase multi-criteria decision-making (TPMCDM) method is presented here to deal with the personnel selection problem. In the proposed method, TOPSIS, preference ranking organization method for enrichment evaluation (PROMETHEE), entropy method, and 2-tuple linguistic variables will be integrated to handle the personnel selection problem.

TOPSIS is one of the famous MCDM methods that deals with decision-making problems [6]. Up to now, TOPSIS has been applied in different management areas such as personnel selection $[7,8]$, project selection [9], weapon selection [10], bridge risk assessment [11], total quality management (TQM) consultant selection [12], etc. The way of thinking of TOPSIS is to determine the ranking order of all alternatives in accordance with the relative distance between the positive ideal and negative ideal alternatives [13]. Brans et al. [14] developed PROMETHEE (preference ranking organization method for enrichment evaluation) to deal with multi-criteria decision-making problems. In the PROMETHEE method, there are six types of preference functions provided for experts to express their opinions with respect to each criterion [15]. This method is also used to deal with many decision-making problems such as personnel selection [16], financial [17,18], and factory location decisions [19].

Different criteria should have different levels of importance in the decision-making process. The entropy method can be used to compute the weight of each criterion effectively [20]. In fact, the entropy method has been widely used in many areas such as engineering [21,22], economic analysis, and finance management [23]. The concept of entropy is an index to measure the uncertainties and degree of messiness of a system [24]. Entropy can provide useful information in accordance with the evaluation data in the decision-making process. For an evaluation criterion, if the difference among the data is low, then the entropy value for this criterion is high. It means that this criterion cannot provide important information for making comparisons among alternatives. Therefore, the weight of this criterion should be low in the decision-making process. On the contrary, if the entropy value of data for this criterion is lower, then the weight of this criterion will be higher than other criteria in the decision-making process [23]. In essence, the entropy method is a systematic way to compute the weight of each criterion based on the evaluation data.

As many qualitative criteria should be considered in the MCDM process, it is suitable for experts or decision-makers to use the linguistic variables to express their opinions. Based on the different backgrounds and experiences of experts, multi-type linguistic variables are suitable for expressing the evaluation values $[25,26]$. The 2-type linguistic variable can express the opinions of decision-makers and make the information translation more effective [27]. Therefore, multi-type linguistic variables based on 2-type linguistic terms are used to express the evaluation values of decision-makers in this paper.

According to the above narrations, this paper is organized into seven sections. Section 2 discusses the literature. Section 3 introduces the definitions and operations of a 2-tuple linguistic variable. Section 4 explains the details of the proposed model. Section 5 presents an example to describe the practicability of the proposed model. Section 6 executes a simulation experiment in order to prove the effectiveness of the proposed model. Finally, Section 7 offers the advantages of the proposed model.

\section{Literature Review}

Ertugrul Karsak [28] used fuzzy objective programming to choose the person. Korvin et al. [29] selected the suitable person by considering the levels of compatibility and acceptable levels of quality 
to compute the fuzzy fitness of each person to do the right jobs. Chien and Chen [30] proposed an effective data mining method based on the rough set theory to select the right person from a human resource database. Gungor et al. [31] considered many quantitative and qualitative factors and used fuzzy analytic hierarchy process (AHP) to cope with the personnel selection problem. According to maritime human resources data, Celik et al. [32] combined fuzzy AHP with fuzzy TOPSIS to deal with the academic personnel selection problem. Some researchers used the objective programming model and machine learning method to deal with the personnel selection problem for a project team [33,34].

Zhang and Liu [35] combined intuitionistic fuzzy numbers with the grey relational method to select suitable engineers for a software company. Chen et al. [36] used the linguistic VIKOR (Vlsekriterijumska Optimizacija I Kompromisno Resenje) method and skill knowledge map to choose new staff in accordance with the job requirement. Bangerter et al. [37] selected a suitable person according to past performance of applicants based on the signaling theory. Afshari et al. [38] applied the fuzzy integral to perform personnel selection when the personnel selection criteria depended on each other. Sang et al. [39] used fuzzy number to evaluate the weight of each criterion and the performance of an applicant. Fuzzy TOPSIS was used to rank the applicants. Compared with the traditional TOPSIS method, their method can maintain computational efficiency to some extent and avoid information loss. Ji et al. [40] considered the risk preference of decision-makers by using a multi-valued linguistic variable to let experts express their opinions. After that, the projection-based iterative multi-criteria decision making (TODIM) method was applied to handle the personnel selection problem. The projection-based TODIM method was compared with some traditional MCDM methods for verifying feasibility of this method.

Heidary Dahooie et al. [41] designed the competency framework with five criteria in order to choose the best information technology expert. Grey additive ratio assessment (ARAS-G) and the stepwise weight assessment ratio analysis (SWARA) were integrated to derive the criteria weights and to determine the final ranking order of all candidates. Yalçın and Yapıcı Pehlivan [42] used the hesitant fuzzy linguistic terms to express the evaluation opinions of experts to deal with the personnel selection problem. In order to justify the usefulness of this method, fuzzy WASPAS (Weighted Aggregated Sum Product Assessment), fuzzy ARAS, fuzzy EDAS (Evaluation based on Distance from Average Solution), fuzzy TOPSIS, and fuzzy WASPAS were used to analyze and evaluate the personnel selection results by using the same data. The experiment result showed that this methodology is stable and efficient for solving personnel selection problems. Chuang et al. [43] designed a data-driven multi-attribute decision-making model. This model applied a machine learning mechanism to reduce the influence by the subjectivity experience of the experts. Rough set theory was used to derive an initial influential significance-relation matrix from real assessment data. A dynamic analytic network process was applied to acquire the influential significance-network relation map and influential weights from the initial matrix. PROMETHEE-AS can then be applied to evaluate the gap between the aspiration and current levels for each applicant. Kilic et al. [44] applied DEMATEL and ELECTRE methods to rank the air-filter manufacturing applicants. Krishankumar et al. [45] used intuitionistic fuzzy set (IFS) to express the opinions of experts and combined VIKOR method with IFS to effectively handle personnel selection problem. Ozdemir and Nalbant [46] used five main criteria for evaluating the applicants in Turkey. They combined consistent fuzzy preference relation (CFPR) with fuzzy analytic hierarchy process (FAHP) to rank the performances of those applicants.

\section{The 2-Tuple Linguistic Variables}

Many quantitative and qualitative factors in general will influence the decision-making of the personnel selection. For quantitative criteria, it is easy to use crisp values to express the evaluation values, such as working years, the grade of a language test such as TOEIC (Test of English for International Communication) grade or TOEFL (Test of English as a Foreign Language) grade, the level of education, and work licenses [1]. However, decision-makers always find it difficult to express their opinions for qualitative criteria, such as professionalism, working ability, leadership, communication 
skill, and emotional steadiness. In order to express the subjective opinions of experts, the 2-tuple linguistic variables are suitable for experts to express their opinions for the qualitative criteria. A 2-tuple linguistic variable can be defined as a triangular fuzzy number [47]. In this study, there are three types of 2-tuple linguistic variables used in the decision-making process. Different types of linguistic variables and the membership functions are shown as Table 1 and Figures $1-3$, respectively.

Table 1. Different types of linguistic variables.

\begin{tabular}{|c|c|c|c|}
\hline Type & & Linguistic Variable & Figure \\
\hline 1 & Performance & $\begin{array}{l}\text { Extremely Poor }\left(s_{0}^{5}\right), \text { Poor }\left(s_{1}^{5}\right), \text { Fair }\left(s_{2}^{5}\right), \text { Good }\left(s_{3}^{5}\right), \text { Extremely } \\
\qquad \begin{array}{r}\text { Good }\left(s_{4}^{5}\right) \\
\text { Extremely Low }\left(s_{0}^{5}\right), \text { Low }\left(s_{1}^{5}\right), \text { Fair }\left(s_{2}^{5}\right), \operatorname{High}\left(s_{3}^{5}\right), \text { Extremely } \\
\text { High }\left(s^{5}\right)\end{array}\end{array}$ & Figure 1 \\
\hline 2 & Performance & $\begin{array}{l}\text { Extremely Poor }\left(s_{0}^{7}\right) \text {, Poor }\left(s_{1}^{7}\right) \text {, Medium Poor }\left(s_{2}^{7}\right) \text {, Fair }\left(s_{3}^{7}\right) \text {, } \\
\quad \text { Medium Good }\left(s_{4}^{7}\right), \text { Good }\left(s_{5}^{7}\right) \text {, Extremely Good }\left(s_{6}^{7}\right) \\
\text { Extremely Low }\left(s_{0}^{7}\right) \text {, Low }\left(s_{1}^{7}\right) \text {, Medium Low }\left(s_{2}^{7}\right) \text {, Fair }\left(s_{3}^{7}\right) \text {, } \\
\quad \text { Medium High }\left(s_{4}^{7}\right), \text { High }\left(s_{5}^{7}\right) \text {, Extremely High }\left(s_{6}^{7}\right)\end{array}$ & Figure 2 \\
\hline 3 & Performance & $\begin{array}{l}\text { Extremely Poor }\left(s_{0}^{9}\right) \text {, Very Poor }\left(s_{1}^{9}\right) \text {, Poor }\left(s_{2}^{9}\right) \text {, Medium Poor } \\
\text { Extremely Good }\left(s_{4}^{9}\right) \text {, Medium Good }\left(s_{5}^{9}\right) \text {, Good }\left(s_{6}^{9}\right) \text {, Very Good }\left(s_{7}^{9}\right) \text {, } \\
\text { Extremely Low }\left(s_{0}^{9}\right) \text {, Very Low }\left(s_{1}^{9}\right) \text {, Low }\left(s_{2}^{9}\right) \text {, Medium Low } \\
\left(s_{3}^{9}\right) \text {, Fair }\left(s_{4}^{9}\right) \text {, Medium High }\left(s_{5}^{9}\right), \operatorname{High}\left(s_{6}^{9}\right) \text {, Very High }\left(s_{7}^{9}\right) \text {, } \\
\text { Extremely High }\left(s_{8}^{9}\right)\end{array}$ & Figure 3 \\
\hline
\end{tabular}

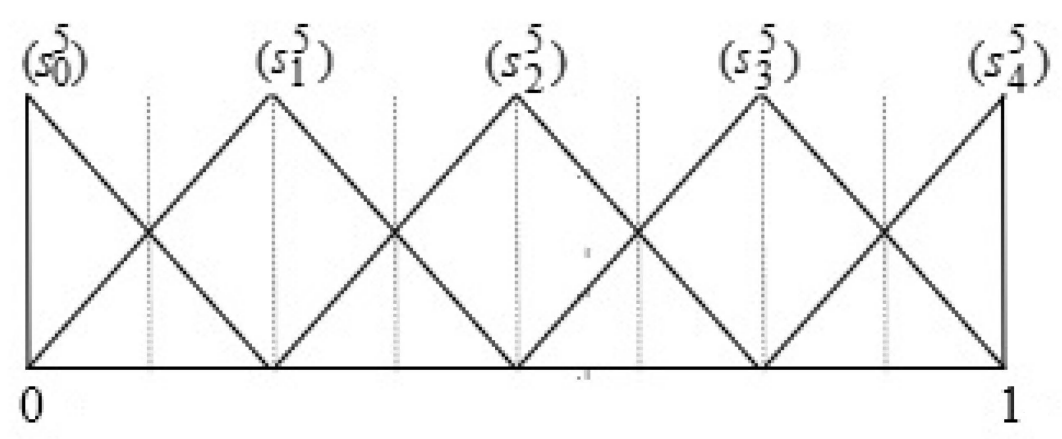

Figure 1. Membership functions of linguistic variables at type 1.

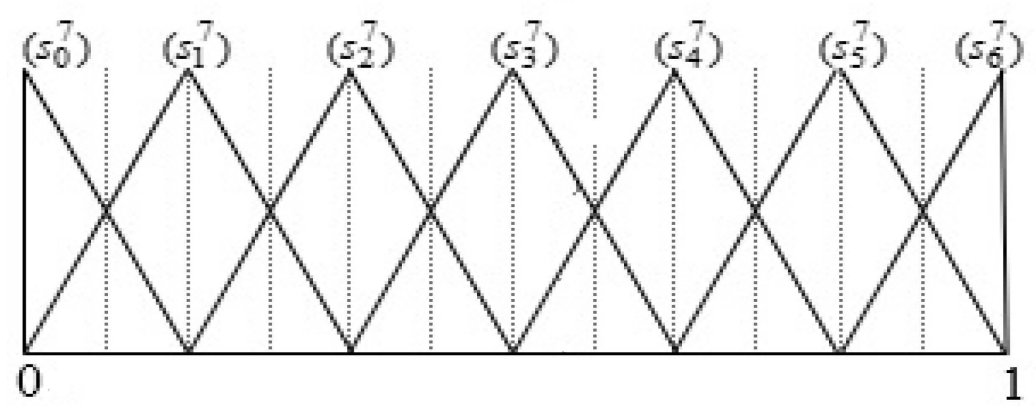

Figure 2. Membership functions of linguistic variables at type 2. 


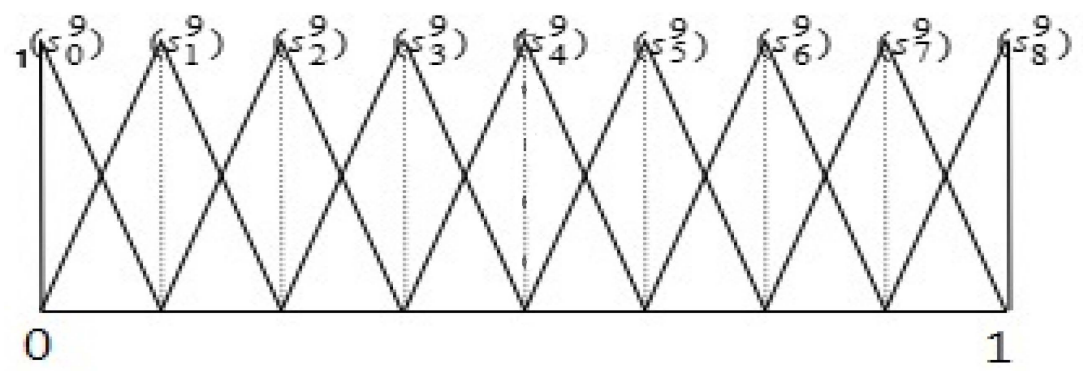

Figure 3. Membership functions of linguistic variables at type 3.

Suppose that $S=\left\{s_{0}, s_{1}, s_{2}, \ldots, s_{\mathrm{g}}\right\}$ is an ordered linguistic term set; $\mathrm{g}$ is the number used to represent the index of maximum linguistic variable in linguistic term set $S ; s_{0}, s_{1}, s_{2}, \ldots, s_{\mathrm{g}}$ are linguistic variables. A 2-tuple linguistic variable $(L)$ can be expressed by two elements as $\left(s_{i}, \alpha_{i}\right)$ in accordance with the linguistic term set. The symbol $s_{i}$ is the central value of the $i$ th linguistic variable in $S$, and symbol $\alpha_{i}$ is the difference in values between $\mathrm{L}$ and the $i$ th linguistic variable in $\mathrm{S}$. Before the operation of 2-tuple linguistic variables, we must transform 2-tuple linguistic variables into crisp values. For the same reason, we can transform a crisp value into a 2-tuple linguistic variable to be expressed as a triangular fuzzy number. Applying the translation function $(\Delta)$, we can easily transform a crisp value $\beta$ into a 2-tuple linguistic variable [47]. In this paper, the generalized translation function can be applied as [48]:

$$
\Delta(\beta)=\left(s_{i}, \alpha_{i}\right),
$$

where $\Delta$ is symbol of translation function, $i=\operatorname{round}(\beta \times \mathrm{g}), \alpha_{i}=\beta-\frac{i}{\mathrm{~g}}, \beta \in[0,1]$ and $\alpha_{i} \in\left[-\frac{1}{2 \mathrm{~g}}, \frac{1}{2 \mathrm{~g}}\right)$.

A reverse function $\Delta^{-1}$ is applied to transform a 2-tuple linguistic information $\left(s_{i}, \alpha_{i}\right)$ into a crisp value. According to the generalized translation function, the crisp value can be computed as follows [48].

$$
\Delta^{-1}\left(s_{i}, \alpha_{i}\right)=\frac{i}{g}+\alpha_{i}
$$

Suppose that $\mathrm{x}=\left\{\left(s_{i}, \alpha_{i}\right), i=1,2, \ldots, n\right\}$ is a set of 2-tuple linguistic variables. The term $\bar{X}$ is the mean value, which is acquired from the set of the 2-tuple linguistic variable $\mathrm{x}=\left\{\left(s_{i}, \alpha_{i}\right), i=1,2, \ldots, n\right\}$. It can be computed as [49]:

$$
\bar{X}=\frac{1}{n} \sum_{i=1}^{n} \Delta^{-1}\left(s_{i}, \alpha_{i}\right)
$$

As a result of different expertise backgrounds, knowledge, and experiences, experts use different types of linguistic terms to express their evaluation values in a decision-making process [50]. For example, if an expert has more knowledge or expertise background for the decision problem, then he/she will choose the type 3 linguistic variables (shown as Table 1) to present a more precise opinion in the decision-making process. To aggregate the opinions of all experts, one needs to transfer these different types of 2-tuple linguistic variables into a standard linguistic set. Herrera and Martinez [47] presented a method to transform different types of 2-tuple linguistic variables into a standard linguistic set, but the transform results cannot keep the same domain in accordance with their transform method. Therefore, a generalized transform method is applied here to transform the different types of linguistic variable into a common linguistic term at the same domain [48,51].

Suppose that the different types of linguistic variable sets can be defined by partitioning the interval $[0,1]$ (shown as Table 1 ). According to the generalized transform method, a crisp value $\beta$ ( $\beta \in$ $[0,1])$ can be transformed into an $i$ th linguistic term $\left(s_{i}^{n(t)}, \alpha_{i}^{n(t)}\right)$ of type $t$ as:

$$
\Delta_{t}(\beta)=\left(s_{i}^{n(t)}, \alpha_{i}^{n(t)}\right)
$$


where $i=\operatorname{round}\left(\beta \times \mathrm{g}_{t}\right), \alpha_{i}^{n(t)}=\beta-\frac{\mathrm{i}}{\mathrm{g}_{t}}, \mathrm{~g}_{t}=n(t)-1$, and $n(t)$ is the number of $t$ th type of linguistic variables. In this research, three kinds of 2-tuple linguistic variables are used. The membership functions of linguistic variables are shown as Figures 1-3. Based on the generalized transform method, we can transform the $i$ th linguistic term of type $t$ into a crisp value $\beta(\beta \in[0,1])$ as:

$$
\Delta_{t}^{-1}\left(s_{i}^{n(t)}, \alpha_{i}^{n(t)}\right)=\frac{i}{g_{t}}+\alpha_{i}^{n(t)}=\beta
$$

where $\mathrm{g}_{t}=n(t)-1$, and $\alpha_{i}^{n(t)} \in\left[-\frac{1}{2 \mathrm{~g}_{t}}, \frac{1}{2 \mathrm{~g}_{t}}\right)$.

We therefore can transform the $i$ th linguistic term $\left(s_{i}^{n(t)}, \alpha_{i}^{n(t)}\right)$ of type $t$ into the $k$ th linguistic term $\left(s_{k}^{n(t+1)}, \alpha_{k}^{n(t+1)}\right)$ of type $t+1$ at the same domain interval $[0,1]$ as:

$$
\Delta_{t+1}\left(\Delta_{t}^{-1}\left(s_{i}^{n(t)}, \alpha_{i}^{n(t)}\right)\right)=\left(s_{k}^{n(t+1)}, \alpha_{k}^{n(t+1)}\right),
$$

where $g_{t+1}=n(t+1)-1$, and $\alpha_{k}^{n(t+1)} \in\left[-\frac{1}{2 g_{t+1}}, \frac{1}{2 g_{t+1}}\right)$.

\section{The TPMCDM Method}

As we know, a personnel selection problem can be regarded as a MCDM problem with multiple experts (or decision-makers). Basically, the problem structure of personnel selection can be divided into five parts by means of the following sets.

(i) A set of experts (decision-makers) is called $\mathrm{E}=\left\{E_{1}, E_{2}, \ldots, E_{k}\right\}$.

(ii) A set of applicants (alternatives) is called $\mathrm{A}=\left\{A_{1}, A_{2}, \ldots, A_{m}\right\}$.

(iii) A set of criteria is called $C=\left\{C_{1}, C_{2}, \ldots, C_{n}\right\}$. The set of criteria can be divided into two subsets such as quantitative set $C^{\text {quan }}=\left\{C_{1}, C_{2}, \ldots, C_{z}\right\}$ and qualitative set $C^{\text {qual }}=\left\{C_{z+1}, C_{z+2}, \ldots, C_{n}\right\}$.

(iv) A weight set of quantitative criteria is called $W^{\text {quan }}=\left\{w_{1}^{\text {quan }}, w_{2}^{\text {quan }}, \ldots, w_{Z}^{\text {quan }}\right\}$ for evaluating the importance of criterion $C_{j}(j=1,2, \ldots, \mathrm{z})$ and $\sum_{i=1}^{z} w_{i}^{\text {quan }}=1$. A weight set of qualitative criteria is called $W^{\text {qual }}=\left\{w_{z+1}^{\text {qual }}, w_{z+2^{\text {qual }}}, \ldots, w_{n}^{\text {qual }}\right\}$ for evaluating the importance of criterion $C_{j}(j=z+1, z+2$, $\ldots, \mathrm{n})$ and $\sum_{i=z+1}^{n} w_{i}^{\text {qual }}=1$.

(v) The crisp performance ratings of applicants with respect to quantitative criteria can be represented as $x_{i j}, i=1,2, \ldots, \mathrm{m}$ and $j=1,2, \ldots, \mathrm{z}$. The linguistic performance ratings of each applicant with respect to qualitative criteria can be described as $\widetilde{x}_{i j}, i=1,2, \ldots$, m and $j=\mathrm{z}+1, \mathrm{z}+2, \ldots, \mathrm{n}$.

According to the definitions of symbols, the decision matrix by aggregating the evaluations of experts can be formulated as:

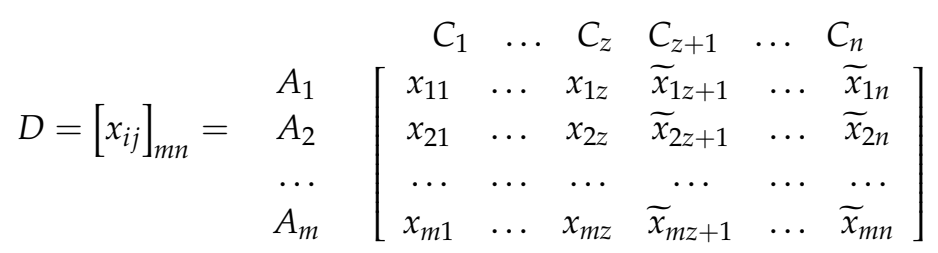

According to the decision matrix, the performance of the $i$ th applicant with respect to the $j$ th criterion by the $k$ th expert can be expressed as a crisp value $\left(x_{i j}^{k}\right)$ for the quantitative criteria $\left(C_{j} \in C^{q u a n}\right)$. The mean value of all $x_{i j}^{k}$ can be represented as $x_{i j}$. The performance of the $i$ th applicant with respect to the $j$ th criterion by the $k$ th expert can be represented as a 2-tuple linguistic variable $\widetilde{x}_{i j}^{k}=\left(s_{i j}^{k}\right.$, $\left.\alpha_{i j}^{k}\right)$ for the quantitative criteria $\left(C_{j} \in C^{q u a l}\right)$. The aggregated linguistic rating $\left(\widetilde{x}_{i j}\right)$ of the $i$ th applicant with respect to the $j$ th criterion can be calculated as: 


$$
\widetilde{x}_{\mathrm{ij}}=\Delta\left(\frac{1}{K} \sum_{k=1}^{K} \Delta^{-1}\left(s_{i j}^{k}, \alpha_{i j}^{k}\right)\right)=\left(s_{i j}, \alpha_{i j}\right)
$$

Formula (8) is used to integrate experts' opinions. The concept of Formula (8) is that an expert's opinion is transferred as a crisp value by Formula (2). The average value of experts' opinions (crisp value type) can be calculated. Finally, experts' integrated opinions (crisp value type) will be transferred as a 2-tuple linguistic variable by Formula (1).

The aim of this paper is to provide the two-phase multiple-criteria decision-making (TPMCDM) method to deal with the personnel selection problem. It can be divided into two phases. In the first stage, the evaluation values of each applicant with respect to quantitative criteria are considered to eliminate some unsuitable applicants by using the TOPSIS method. In the second stage, the different preference functions of the qualitative criteria are applied to evaluate the suitable candidates by using the PROMETHEE method. By considering the quantitative and qualitative criteria simultaneously, a comprehensive support index is defined to determine the ranking order of all suitable candidates in accordance with the closeness coefficients of TOPSIS and outranking indices of PROMETHEE simultaneously. The whole process of the TPMCDM method is shown in Figure 4.

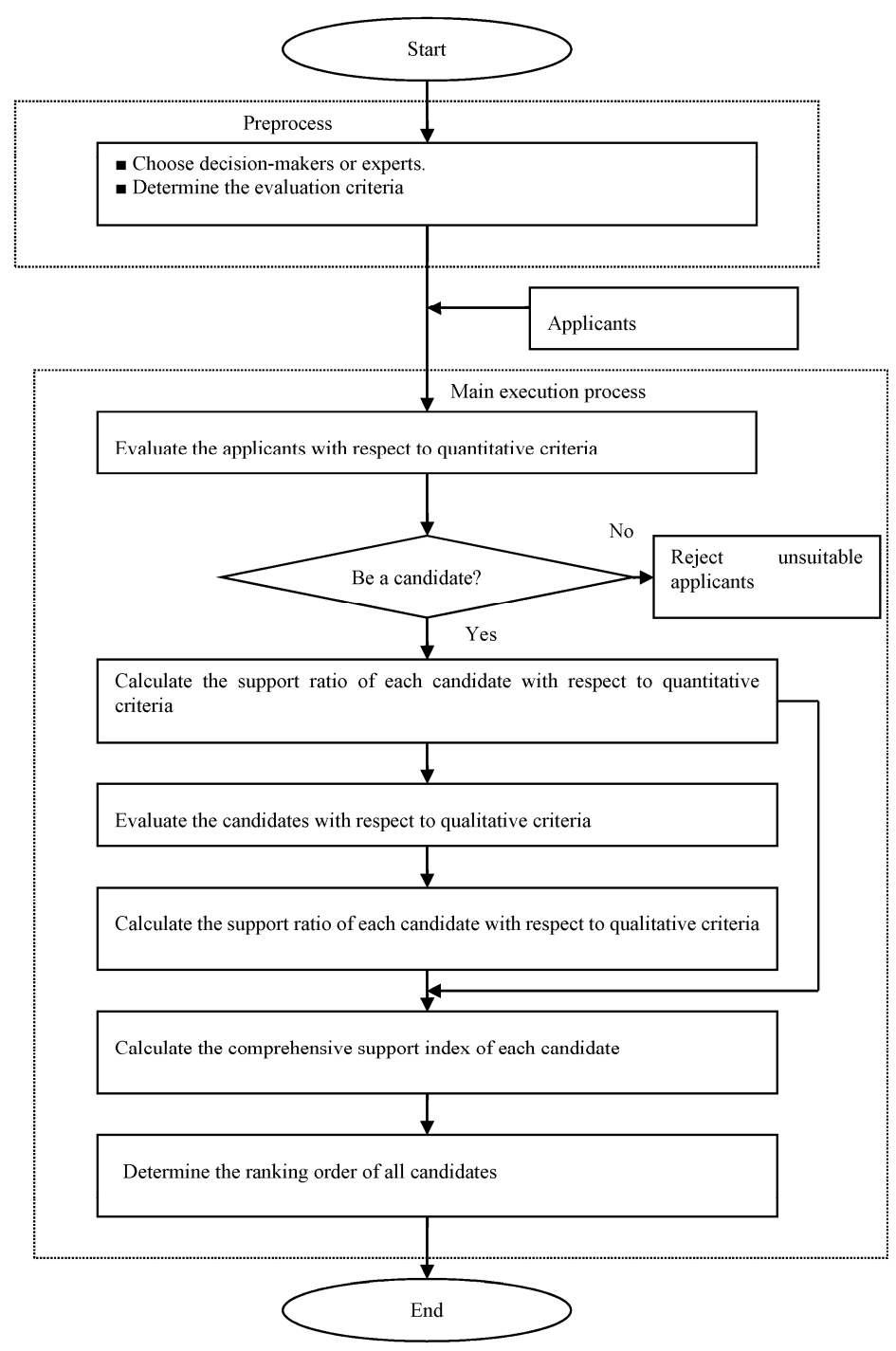

Figure 4. The process of two-phase multi-criteria decision-making (MCDM) method for personnel selection. 


\subsection{The First Phase}

In order to compare the performance ratings of applicants with respect to quantitative criteria more objectively, the performance ratings of applicants can be normalized as:

$$
t_{i j}=\frac{x_{i j}}{\sqrt{\sum_{i=1}^{m} x_{i j}^{2}}}, i=1, \ldots, m j=1, \ldots, z
$$

The entropy value of the $j$ th quantitative criterion $\left(e_{j}^{q u a n}\right)$ can be computed as:

$$
e_{j}^{q u a n}=-\frac{1}{\ln m} \sum_{i=1}^{m} t_{i j} \ln t_{i j} i=1, \ldots, m j=1, \ldots, z
$$

Therefore, the weight of the $j$ th quantitative criterion $\left(\mathrm{w}_{j}^{\text {quan }}\right)$ can be computed as:

$$
\mathrm{w}_{j}^{\text {quan }}=\frac{1-e_{j}^{\text {quan }}}{\sum_{j=1}^{z} 1-e_{j}^{\text {quan }}} i=1, \ldots, m j=1, \ldots, z
$$

We construct the weighted normalized decision matrix as:

$$
V=\left[v_{i j}\right], \quad i=1, \ldots, m \quad j=1, \ldots, z,
$$

where $v_{i j}=t_{i j} * \mathrm{w}_{j}^{\text {quan }}$.

According to the weighted normalized decision matrix, the positive-ideal solution (PIS, $\mathrm{A}^{*}$ ) and negative-ideal solution (NIS, $\left.\mathrm{A}^{-}\right)$can be defined as $\mathrm{A}^{*}=\left(v_{1}^{*}, v_{2}^{*}, \ldots, v_{Z}^{*}\right)$ and $\mathrm{A}^{-}=\left(v_{1}^{-}, v_{2}^{-}, \ldots, v_{\mathrm{Z}}^{-}\right)$, where $v_{j}^{*}=\max _{i}\left\{v_{i j}\right\}$ and $v_{j}^{-}=\min _{i}\left\{v_{i j}\right\}$.

The distance of each applicant $A_{i}(i=1,2, \ldots, m)$ from $\mathrm{A}^{*}$ and $\mathrm{A}^{-}$can be currently calculated as:

$$
\begin{aligned}
& d_{i}^{*}=d\left(\mathrm{~A}_{i}, \mathrm{~A}^{*}\right)=\sqrt{\sum_{j=1}^{z}\left(v_{j}^{+}-v_{i j}\right)^{2}} \\
& d_{i}^{-}=d\left(\mathrm{~A}_{i}, \mathrm{~A}^{-}\right)=\sqrt{\sum_{j=1}^{z}\left(v_{i j}-v_{j}^{-}\right)^{2}}
\end{aligned}
$$

The closeness coefficient $\left(C C\left(A_{i}\right)\right)$ of each applicant is calculated as:

$$
\operatorname{CC}\left(A_{i}\right)=\frac{d_{i}^{-}}{d_{i}^{*}+d_{i}^{-}}
$$

where $0 \leq C C\left(A_{i}\right) \leq 1$.

If $C C\left(A_{i}\right)=1$, then $A_{i}$ is the best applicant. If $C C\left(A_{i}\right)=0$, then $A_{i}$ is the worst applicant. If $C C\left(A_{i}\right) \geq C C\left(A_{j}\right)$, then applicant $A_{i}$ is preferred to $A_{j}$. Therefore, we can determine the ranking order of all applicants in accordance with closeness coefficients.

From the closeness coefficients of all applicants, we choose some suitable applicants to evaluate in accordance with their ranking order. If we set the elimination ratio as $R_{e}$, then the number of qualified applicants can be determined as:

$$
G_{b}=\left\{A_{i} \mid \operatorname{Rank}\left(A_{i}\right) \leq \operatorname{Round}\left(m *\left(1-R_{e}\right)\right)\right\},
$$


where $G_{b}$ represents the set of qualified applicants. $\operatorname{Rank}\left(A_{i}\right)$ represents the rank of applicant $A_{i}$ in accordance with the closeness coefficient. $\operatorname{Round}\left(m *\left(1-R_{e}\right)\right)$ represents the number of applicants needed to be evaluated at the next stage.

According to the closeness coefficients of qualified applicants, a support ratio of each qualified applicant with respect to quantitative criteria can be defined as:

$$
\operatorname{SR}_{\text {quan }}\left(A_{i}\right)=\frac{C C\left(A_{i}\right)}{\sum_{A_{i} \in G_{b}} C C\left(A_{i}\right)},
$$

where $0 \leq S R_{\text {quan }}\left(A_{i}\right) \leq 1$.

\subsection{The Second Phase}

At the second stage, we evaluate the qualified applicants (suitable applicants or candidates) in accordance with qualitative criteria. For any two suitable applicants $A_{i}$ and $A_{S}\left(A_{i}, A_{S} \in \mathrm{G}_{\mathrm{b}}\right)$, the difference value between them with respect to qualitative criterion $j$ can be expressed as $d_{j}^{\text {is }}=\Delta^{-1}\left(\widetilde{x}_{\mathrm{ij}}\right)-\Delta^{-1}\left(\widetilde{x}_{\mathrm{sj}}\right)$. Therefore, a preference degree of suitable applicant $A_{i}$ over $A_{S}$ for qualitative criterion $\mathrm{j}$ is defined as:

$$
P_{j}\left(A_{i}, A_{s}\right)=H_{\mathrm{j}}\left(\widetilde{x}_{\mathrm{ij}}, \widetilde{x}_{\mathrm{sj}}\right)=H_{j}\left(d_{j}^{i s}\right),
$$

where $0 \leq P_{j}\left(A_{i}, A_{s}\right) \leq 1$. The symbol $P_{j}\left(A_{i}, A_{s}\right)$ represents the preference degree of suitable applicant $A_{i}$ over $A_{S}$ with respect to the $j$ th criterion. Here, $H_{j}(*)$ is a preference function of the $j$ th criterion.

For dealing with qualitative information, we express the criterion with a linear preference and indifference function (shown in Figure 5) as:

$$
H_{\mathrm{j}}\left(\widetilde{x}_{\mathrm{ij}}, \widetilde{\mathrm{x}}_{\mathrm{sj}}\right)=\left\{\begin{array}{c}
1, \Delta^{-1}\left(\widetilde{x}_{\mathrm{ij}}\right)-\Delta^{-1}\left(\widetilde{x}_{\mathrm{sj}}\right) \geq p \\
\frac{\Delta^{-1}\left(\widetilde{x}_{\mathrm{ij}}\right)-\Delta^{-1}\left(\widetilde{x}_{\mathrm{sj}}\right)-q}{p-q}, q<\Delta^{-1}\left(\widetilde{x}_{\mathrm{ij}}\right)-\Delta^{-1}\left(\widetilde{x}_{\mathrm{sj}}\right)<p \\
0, \Delta^{-1}\left(\widetilde{x}_{\mathrm{ij}}\right)-\Delta^{-1}\left(\widetilde{x}_{\mathrm{sj}}\right) \leq \mathrm{q}
\end{array}\right.
$$

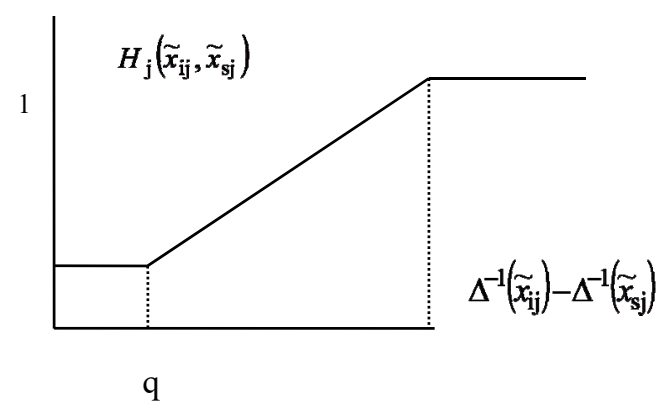

Figure 5. Criterion with linear preference and indifference function.

Here, $\mathrm{p}$ and $\mathrm{q}$ are threshold values, $\widetilde{x}_{i j}$ and $\widetilde{x}_{s j}$ are two-tuple linguistic variable, and $\Delta^{-1}$ is the function that transforms 2-tuple linguistic information into a crisp value.

Level criterion with a linear preference function (shown in Figure 6) for qualitative information is expressed as:

$$
H_{j}\left(\widetilde{x}_{\mathrm{ij}}, \widetilde{x}_{\mathrm{sj}}\right)=\left\{\begin{array}{cc}
1, & \Delta^{-1}\left(\widetilde{x}_{\mathrm{ij}}\right)-\Delta^{-1}\left(\widetilde{x}_{\mathrm{sj}}\right) \geq p \\
\frac{1}{2}, & q<\Delta^{-1}\left(\widetilde{x}_{\mathrm{ij}}\right)-\Delta^{-1}\left(\widetilde{x}_{\mathrm{sj}}\right)<p \\
0, & \Delta^{-1}\left(\widetilde{x}_{\mathrm{ij}}\right)-\Delta^{-1}\left(\widetilde{x}_{\mathrm{sj}}\right) \leq \mathrm{q}
\end{array}\right.
$$




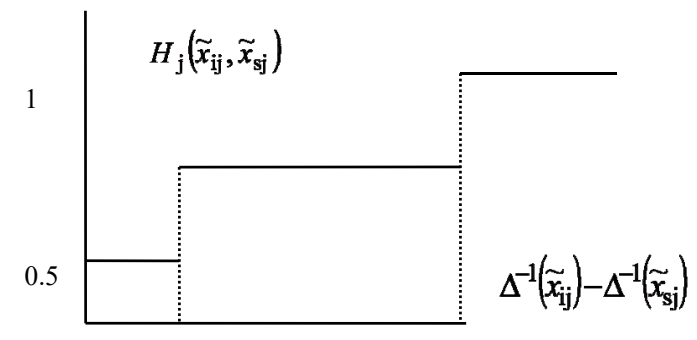

q

Figure 6. Level criterion with linear preference function.

The entropy value of the $j$ th qualitative criterion $\left(e_{j}^{q u a l}\right)$ can be computed as:

$$
e_{j}^{q u a l}=-\frac{1}{\ln \left|G_{b}\right|} \sum_{A_{i} \in G_{b}} \Delta^{-1}\left(\widetilde{x}_{\mathrm{ij}}\right) * \ln \left(\Delta^{-1}\left(\widetilde{x}_{\mathrm{ij}}\right)\right), i=1, \ldots,\left|G_{b}\right| j=z+1, \ldots, n
$$

Here, $\left|G_{b}\right|$ is the number of elements in set $G_{b}$.

The weight of the $j$ th qualitative criterion $\left(\mathrm{w}_{j}^{q u a l}\right)$ can be computed as:

$$
\mathrm{w}_{j}^{q u a l}=\frac{1-e_{j}^{q u a l}}{\sum_{j=z+1}^{n}\left(1-e_{j}^{q u a l}\right)} i=1, \ldots,\left|G_{b}\right| j=z+1, \ldots, n
$$

The overall preference index of suitable applicant $A_{i}$ over $A_{s}$ can be represented as:

$$
\pi\left(A_{i}, A_{s}\right)=\sum_{j=z+1}^{n} \mathrm{w}_{j}^{q u a l} * H_{j}\left(d_{\mathrm{j}}^{\text {is }}\right)
$$

The leaving flow of $A_{i}$ can be calculated as:

$$
\varphi^{+}\left(A_{i}\right)=\sum_{A_{s} \in G_{b}} \pi\left(A_{i}, A_{s}\right)
$$

where $\varphi^{+}\left(A_{i}\right)$ is the dominating degree of $A_{i}$ over the other suitable applicants.

The entering flow of $A_{i}$ can be calculated as:

$$
\varphi^{-}\left(A_{i}\right)=\sum_{A_{s} \in G_{b}} \pi\left(A_{s}, A_{i}\right)
$$

where $\varphi^{-}\left(A_{i}\right)$ is the dominating degree of $A_{i}$ by the other suitable applicants.

The net flow of $A_{i}$ can be calculated as:

$$
\varphi\left(A_{i}\right)=\varphi^{+}\left(A_{i}\right)-\varphi^{-}\left(A_{i}\right)
$$

We define the outranking index of candidate $A_{i}$ as:

$$
\operatorname{OTI}\left(A_{i}\right)=\frac{\frac{\varphi\left(A_{i}\right)}{\left|G_{b}\right|-1}+1}{2},
$$

where $0 \leq \operatorname{OTI}\left(A_{i}\right) \leq 1$, and $A_{i} \in G_{b}$. 
According to the outranking index of each suitable applicant by using the PROMETHEE method, a support ratio of each suitable applicant with respect to qualitative criteria is calculated as:

$$
S R_{\text {qual }}\left(A_{i}\right)=\frac{\operatorname{OTI}\left(A_{i}\right)}{\sum_{A_{i} \in G_{b}} \operatorname{OTI}\left(A_{i}\right)},
$$

where $0 \leq S R_{\text {qual }}\left(A_{i}\right) \leq 1$.

We can therefore define a comprehensive support index of each suitable applicant by considering the quantitative and qualitative criteria simultaneously as:

$$
\operatorname{SR}_{\text {comp }}\left(A_{i}\right)=D_{P} *\left(\operatorname{SR}_{\text {quan }}\left(A_{i}\right)\right)+\left(1-D_{p}\right) *\left(\operatorname{SR}_{\text {qual }}\left(A_{i}\right)\right),
$$

where $D_{P}$ is the preference index of the final decision-maker, and $0 \leq D_{P} \leq 1$.

If $D_{P}=1$, then it means that the decision-maker selects a qualified applicant according to quantitative information. If $D_{P}=0$, then it means that the decision-maker depends on qualitative information to select the qualified applicants. The higher the comprehensive support index $\operatorname{SR}_{\text {comp }}\left(A_{i}\right)$ is, the better suitable applicant $A_{i}$ is. Finally, the ranking order of all suitable applicants in $G_{b}$ can be determined in accordance with the comprehensive support indices.

\section{Numerical Example}

In order to illustrate the computational process of the two-phase multiple-criteria decision-making (TPMCDM) method for the personnel selection clearly, a selection problem of an overseas marketing manager is shown in this section. A decision-making committee consists of three experts to choose the best overseas marketing manager from twenty applicants. The influence factors of personnel selection were first collected from the literatures and a questionnaire was designed for asking managers of enterprises to obtain the evaluation criteria. There are eight criteria (shown as Table 2) used to evaluate the applicants. Quantitative criteria include English ability $\left(C_{1}\right)$, educational background $\left(C_{2}\right)$, work experience $\left(C_{3}\right)$, and license $\left(C_{4}\right)$. Qualitative criteria include communication skill $\left(C_{5}\right)$, innovation ability $\left(C_{6}\right)$, advertise design ability $\left(C_{7}\right)$, and emotional steadiness $\left(C_{8}\right)$. According to the TPMCDM method, the computational steps of this selection problem are summarized as follows.

Table 2. The description of each criterion.

\begin{tabular}{lll}
\hline \multicolumn{1}{c}{ Criterion } & Data Type & \multicolumn{1}{c}{ Preference Function } \\
\hline$C_{1}$ Language ability (TOEIC) & Quantitative & Criterion with linear preference and indifference area \\
$C_{2}$ Educational background & Quantitative & Level criterion with linear preference \\
$C_{3}$ Work experience (Year) & Quantitative & Level criterion with linear preference \\
$C_{4}$ License & Quantitative & Criterion with linear preference and indifference area \\
$C_{5}$ Communication skill & Qualitative & Criterion with linear preference and indifference area \\
$C_{6}$ Innovation ability & Qualitative & Level criterion with linear preference \\
$C_{7}$ Advertise design ability & Qualitative & Criterion with linear preference and indifference area \\
$C_{8}$ Emotional steadiness & Qualitative & Level criterion with linear preference \\
\hline
\end{tabular}

\subsection{The Computational Steps Based on Quantitative Criteria}

Step 1. The quantitative data of the twenty applicants are shown as Table 3. English ability $\left(C_{1}\right)$ is represented by TOEIC grade. Educational background $\left(C_{2}\right)$ means the education degree of each applicant. Work experience $\left(C_{3}\right)$ is represented by the length of working time (years). License $\left(C_{4}\right)$ means the number of professional licenses that each applicant possesses. 
Table 3. Quantitative information of each applicant.

\begin{tabular}{ccccccccccc}
\hline & $A_{\mathbf{1}}$ & $\boldsymbol{A}_{\mathbf{2}}$ & $\boldsymbol{A}_{\mathbf{3}}$ & $\boldsymbol{A}_{\mathbf{4}}$ & $\boldsymbol{A}_{\mathbf{5}}$ & $\boldsymbol{A}_{\mathbf{6}}$ & $\boldsymbol{A}_{\mathbf{7}}$ & $\boldsymbol{A}_{\mathbf{8}}$ & $\boldsymbol{A}_{\mathbf{9}}$ & $\boldsymbol{A}_{\mathbf{1 0}}$ \\
\hline$C_{1}$ & 815 & 280 & 220 & 550 & 290 & 870 & 450 & 840 & 150 & 330 \\
$C_{2}$ & 1 & 8 & 1 & 6 & 2 & 5 & 6 & 4 & 3 & 7 \\
$C_{3}$ & 3 & 4 & 3 & 4 & 1 & 4 & 3 & 2 & 3 & 1 \\
$C_{4}$ & 2 & 7 & 3 & 4 & 5 & 6 & 3 & 2 & 7 & 4 \\
\hline & $\boldsymbol{A}_{\mathbf{1 1}}$ & $\boldsymbol{A}_{\mathbf{1 2}}$ & $\boldsymbol{A}_{\mathbf{1 3}}$ & $\boldsymbol{A}_{\mathbf{1 4}}$ & $\boldsymbol{A}_{\mathbf{1 5}}$ & $\boldsymbol{A}_{\mathbf{1 6}}$ & $\boldsymbol{A}_{\mathbf{1 7}}$ & $\boldsymbol{A}_{\mathbf{1 8}}$ & $\boldsymbol{A}_{\mathbf{1 9}}$ & $\boldsymbol{A}_{\mathbf{2 0}}$ \\
\hline$C_{1}$ & 660 & 330 & 450 & 480 & 640 & 220 & 280 & 600 & 720 & 440 \\
$C_{2}$ & 1 & 5 & 7 & 10 & 3 & 4 & 5 & 6 & 7 & 2 \\
$C_{3}$ & 2 & 4 & 2 & 3 & 2 & 1 & 4 & 2 & 3 & 2 \\
$C_{4}$ & 8 & 8 & 4 & 10 & 4 & 5 & 1 & 7 & 2 & 1 \\
\hline
\end{tabular}

Step 2. Based on Table 3, calculate the normalized values and the weights of quantitative criteria by using the entropy method as Table 4.

Table 4. The weight of each quantitative criterion.

\begin{tabular}{cccc}
\hline$w_{1}$ & $w_{2}$ & $w_{3}$ & $w_{4}$ \\
\hline 0.243 & 0.243 & 0.260 & 0.243 \\
\hline
\end{tabular}

Step 3. Calculate the weighted normalized matrix and determine the positive ideal solution $\left(\mathrm{A}^{*}\right.$, PIS) and the negative ideal solution ( $\mathrm{A}^{-}$, NIS).

Step 4. For each applicant, the distances from PIS and NIS as well as the closeness coefficient (CC) can be computed as Table 5 .

Table 5. The distance measurement, the closeness coefficient and support rations.

\begin{tabular}{|c|c|c|c|c|c|c|c|c|c|c|}
\hline & $A_{1}$ & $A_{2}$ & $A_{3}$ & $A_{4}$ & $A_{5}$ & $A_{6}$ & $A_{7}$ & $A_{8}$ & $A_{9}$ & $A_{10}$ \\
\hline $\mathrm{d}\left(\mathrm{A}_{\mathrm{i}}, \mathrm{A}^{*}\right)$ & 0.1259 & 0.0736 & 0.1383 & 0.0819 & 0.1309 & 0.0660 & 0.0968 & 0.1109 & 0.1122 & 0.1093 \\
\hline $\mathrm{d}\left(\mathrm{A}_{\mathrm{i}}, \mathrm{A}^{-}\right)$ & 0.0832 & 0.1140 & 0.0465 & 0.0962 & 0.0451 & 0.1190 & 0.0762 & 0.0837 & 0.0770 & 0.0718 \\
\hline $\mathrm{CC}\left(\mathrm{A}_{\mathrm{i}}\right)$ & 0.3979 & 0.6077 & 0.2518 & 0.5401 & 0.2562 & 0.6433 & 0.4404 & 0.4300 & 0.4070 & 0.3963 \\
\hline \multirow[t]{2}{*}{$S R_{\text {quan }}\left(A_{i}\right)$} & - & 0.1092 & - & 0.0971 & 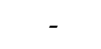 & 0.1156 & 0.0791 & - & - & - \\
\hline & $A_{11}$ & $A_{12}$ & $A_{13}$ & $A_{14}$ & $A_{15}$ & $A_{16}$ & $A_{17}$ & $A_{18}$ & $A_{19}$ & $A_{20}$ \\
\hline $\mathrm{d}\left(\mathrm{A}_{\mathrm{i}}, \mathrm{A}^{*}\right)$ & 0.1059 & 0.0804 & 0.0922 & 0.0467 & 0.1064 & 0.1231 & 0.1237 & 0.0720 & 0.0918 & 0.1386 \\
\hline $\mathrm{d}\left(\mathrm{A}_{\mathrm{i}}, \mathrm{A}^{-}\right)$ & 0.0930 & 0.1052 & 0.0790 & 0.1419 & 0.0677 & 0.0521 & 0.0754 & 0.0961 & 0.0968 & 0.0388 \\
\hline $\mathrm{CC}\left(\mathrm{A}_{\mathrm{i}}\right)$ & 0.4676 & 0.5668 & 0.4613 & 0.7522 & 0.3890 & 0.2972 & 0.3786 & 0.5719 & 0.5132 & 0.2185 \\
\hline$S R_{\text {quan }}\left(A_{i}\right)$ & 0.0840 & 0.1019 & 0.0829 & 0.1352 & - & - & - & 0.1028 & 0.0922 & - \\
\hline
\end{tabular}

Step 5. Supposing that the elimination rate is 50\%, ten qualified applicants are selected in accordance with closeness coefficient values of all applicants as Table 5. Therefore, the set of qualified applicants is $\left\{A_{2}, A_{4}, A_{6}, A_{7}, A_{11}, A_{12}, A_{13}, A_{14}, A_{18}, A_{19}\right\}$.

Step 6. The support ratios of ten qualified applicants based on quantitative criteria can be calculated as Table 5 .

\subsection{The Computational Steps of the Final Ranking Order}

Step 1. Each expert chooses the linguistic variable type to express his/her opinion. In this paper, expert $E_{1}$ chooses type $1, E_{2}$ chooses type 2 , and $E_{3}$ chooses type 3 . The definitions of linguistic variables are shown as Table 1. The membership functions of linguistic variables are shown as Figures 1-3.

Step 2. Three experts use the linguistic variables to express their performance ratings of each qualified applicant with respect to qualitative criteria as Table 6. 
Table 6. The linguistic ratings are evaluated by experts.

\begin{tabular}{|c|c|c|c|c|c|c|c|c|c|}
\hline Criterion & Candidate & $\mathrm{E}_{1}$ & $E_{2}$ & $E_{3}$ & Criterion & Candidate & $\mathrm{E}_{1}$ & $E_{2}$ & $E_{3}$ \\
\hline \multirow{9}{*}{$C_{5}$} & $A_{2}$ & $\left(s_{4}^{5}, 0\right)$ & $\left(s_{6}^{7}, 0\right)$ & $\left(s_{5}^{9}, 0\right)$ & \multirow{9}{*}{$C_{7}$} & $A_{2}$ & $\left(s_{4}^{5}, 0\right)$ & $\left(s_{3}^{7}, 0\right)$ & $\left(s_{5}^{9}, 0\right)$ \\
\hline & $A_{4}$ & $\left(s_{3}^{5}, 0\right)$ & $\left(s_{4}^{7}, 0\right)$ & $\left(s_{5}^{9}, 0\right)$ & & $A_{4}$ & $\left(s_{2}^{5}, 0\right)$ & $\left(s_{6}^{7}, 0\right)$ & $\left(s_{8}^{9}, 0\right)$ \\
\hline & $A_{6}$ & $\left(s_{1}^{5}, 0\right)$ & $\left(s_{5}^{7}, 0\right)$ & $\left(s_{8}^{9}, 0\right)$ & & $A_{6}$ & $\left(s_{3}^{5}, 0\right)$ & $\left(s_{6}^{7}, 0\right)$ & $\left(s_{7}^{9}, 0\right)$ \\
\hline & $A_{7}$ & $\left(s_{2}^{5}, 0\right)$ & $\left(s_{6}^{7}, 0\right)$ & $\left(s_{8}^{9}, 0\right)$ & & $A_{7}$ & $\left(s_{0}^{5}, 0\right)$ & $\left(s_{5}^{7}, 0\right)$ & $\left(s_{5}^{9}, 0\right)$ \\
\hline & $A_{11}$ & $\left(s_{0}^{5}, 0\right)$ & $\left(s_{5}^{7}, 0\right)$ & $\left(s_{7}^{9}, 0\right)$ & & $A_{11}$ & $\left(s_{0}^{5}, 0\right)$ & $\left(s_{3}^{7}, 0\right)$ & $\left(s_{4}^{9}, 0\right)$ \\
\hline & $A_{12}$ & $\left(s_{2}^{5}, 0\right)$ & $\left(s_{6}^{7}, 0\right)$ & $\left(s_{4}^{9}, 0\right)$ & & $A_{12}$ & $\left(s_{4}^{5}, 0\right)$ & $\left(s_{6}^{7}, 0\right)$ & $\left(s_{6}^{9}, 0\right)$ \\
\hline & $A_{13}$ & $\left(s_{4}^{5}, 0\right)$ & $\left(s_{5}^{9}, 0\right)$ & $\left(s_{8}^{9}, 0\right)$ & & $A_{13}$ & $\left(s_{1}^{5}, 0\right)$ & $\left(s_{6}^{q}, 0\right)$ & $\left(s_{7}^{9}, 0\right)$ \\
\hline & $A_{14}$ & $\left(s_{4}^{5}, 0\right)$ & $\left(s_{5}^{7}, 0\right)$ & $\left(s_{8}^{9}, 0\right)$ & & $A_{14}$ & $\left(s_{4}^{5}, 0\right)$ & $\left(s_{3}^{7}, 0\right)$ & $\left(s_{4}^{9}, 0\right)$ \\
\hline & $A_{18}$ & $\left(s_{4}^{5}, 0\right)$ & $\left(s_{3}^{7}, 0\right)$ & $\left(s_{6}^{9}, 0\right)$ & & $A_{18}$ & $\left(s_{3}^{5}, 0\right)$ & $\left(s_{5}^{7}, 0\right)$ & $\left(s_{5}^{9}, 0\right)$ \\
\hline \multirow{11}{*}{$C_{6}$} & $A_{19}$ & $\left(s_{4}^{5}, 0\right)$ & $\left(s_{5}^{7}, 0\right)$ & $\left(s_{5}^{9}, 0\right)$ & \multirow{11}{*}{$C_{8}$} & $A_{19}$ & $\left(s_{4}^{5}, 0\right)$ & $\left(s_{6}^{7}, 0\right)$ & $\left(s_{8}^{9}, 0\right)$ \\
\hline & $A_{2}$ & $\left(s_{4}^{5}, 0\right)$ & $\left(s_{1}^{7}, 0\right)$ & $\left(s_{7}^{9}, 0\right)$ & & $A_{2}$ & $\left(s_{4}^{5}, 0\right)$ & $\left(s_{4}^{7}, 0\right)$ & $\left(s_{6}^{9}, 0\right)$ \\
\hline & $A_{4}$ & $\left(s_{2}^{5}, 0\right)$ & $\left(s_{6}^{7}, 0\right)$ & $\left(s_{7}^{9}, 0\right)$ & & $A_{4}$ & $\left(s_{3}^{5}, 0\right)$ & $\left(s_{6^{7}}^{7}, 0\right)$ & $\left(s_{8}^{9}, 0\right)$ \\
\hline & $A_{6}$ & $\left(s_{2}^{5}, 0\right)$ & $\left(s_{3}^{0}, 0\right)$ & $\left(s_{6}^{9}, 0\right)$ & & $A_{6}$ & $\left(s_{1}^{5}, 0\right)$ & $\left(s_{2}^{0}, 0\right)$ & $\left(s_{8}^{9}, 0\right)$ \\
\hline & $A_{7}$ & $\left(s_{0}^{5}, 0\right)$ & $\left(s_{4}^{7}, 0\right)$ & $\left(s_{8}^{9}, 0\right)$ & & $A_{7}$ & $\left(s_{4}^{5}, 0\right)$ & $\left(s_{6}^{7}, 0\right)$ & $\left(s_{7}^{9}, 0\right)$ \\
\hline & $A_{11}$ & $\left(s_{3}^{5}, 0\right)$ & $\left(s_{4}^{7}, 0\right)$ & $\left(s_{5}^{9}, 0\right)$ & & $A_{11}$ & $\left(s_{2}^{5}, 0\right)$ & $\left(s_{3}^{0}, 0\right)$ & $\left(s_{4^{\prime}}^{9}, 0\right)$ \\
\hline & $A_{12}$ & $\left(s_{4^{\prime}}^{5}, 0\right)$ & $\left(s_{5}^{7}, 0\right)$ & $\left(s_{8}^{9}, 0\right)$ & & $A_{12}$ & $\left(s_{3}^{5}, 0\right)$ & $\left(s_{6^{\prime}}^{7}, 0\right)$ & $\left(s_{5}^{9}, 0\right)$ \\
\hline & $A_{13}$ & $\left(s_{4^{\prime}}^{5}, 0\right)$ & $\left(s_{6}^{7}, 0\right)$ & $\left(s_{5}^{9}, 0\right)$ & & $A_{13}$ & $\left(s_{4}^{5}, 0\right)$ & $\left(s_{3}^{7}, 0\right)$ & $\left(s_{5}^{9}, 0\right)$ \\
\hline & $A_{14}$ & $\left(s_{2}^{5}, 0\right)$ & $\left(s_{3}^{0}, 0\right)$ & $\left(s_{4}^{9}, 0\right)$ & & $A_{14}$ & $\left(s_{0}^{5}, 0\right)$ & $\left(s_{5}^{7}, 0\right)$ & $\left(s_{7}^{9}, 0\right)$ \\
\hline & $A_{18}$ & $\left(s_{4}^{5}, 0\right)$ & $\left(s_{6}^{7}, 0\right)$ & $\left(s_{7}^{9}, 0\right)$ & & $A_{18}$ & $\left(s_{3}^{5}, 0\right)$ & $\left(s_{6}^{7}, 0\right)$ & $\left(s_{4}^{9}, 0\right)$ \\
\hline & $A_{19}$ & $\left(s_{0}^{5}, 0\right)$ & $\left(s_{2}^{q}, 0\right)$ & $\left(s_{5}^{9}, 0\right)$ & & $A_{19}$ & $\left(s_{2}^{5}, 0\right)$ & $\left(s_{4^{\prime}}^{q}, 0\right)$ & $\left(s_{5}^{9}, 0\right)$ \\
\hline
\end{tabular}

Step 3. Transform the different types of linguistic ratings of three experts into type 2. Aggregate the linguistic ratings of each qualified applicant with respect to qualitative criteria.

Step 4. Transform the aggregation linguistic ratings into crisp values and calculate the weights of qualitative criteria by using the entropy method as Table 7.

Table 7. The weight of each qualitative criterion.

\begin{tabular}{ccccc}
\hline & $C_{5}$ & $C_{6}$ & $C_{7}$ & $C_{8}$ \\
\hline Weight & 0.2190 & 0.2674 & 0.2486 & 0.2650 \\
\hline
\end{tabular}

Step 5. Determine the threshold values of preference functions for each qualitative criterion as Table 8 .

Table 8. The threshold values of preference function with respect to each criterion.

\begin{tabular}{ccccccccc}
\hline & $C_{1}$ & $C_{2}$ & $C_{3}$ & $C_{4}$ & $C_{5}$ & $C_{6}$ & $C_{7}$ & $C_{8}$ \\
\hline \multirow{2}{*}{ Threshold values } & $\mathrm{p}=200$ & $\mathrm{p}=2$ & $\mathrm{p}=3$ & $\mathrm{p}=3$ & $\mathrm{p}=1 / 6$ & $\mathrm{p}=1 / 6$ & $\mathrm{p}=1 / 6$ & $\mathrm{p}=1 / 6$ \\
& $\mathrm{q}=100$ & $\mathrm{q}=1$ & $\mathrm{q}=1$ & $\mathrm{q}=1$ & $\mathrm{q}=1 / 12$ & $\mathrm{q}=1 / 12$ & $\mathrm{q}=1 / 12$ & $\mathrm{q}=1 / 12$ \\
\hline
\end{tabular}

Step 6. Calculate the overall preference indices between a pair of qualified applicants.

Step 7. According to PROMETHEE, the leaving flow, the entering flow, the net flow, and the outranking index of each qualified applicant can be calculated as Table 9. 
Table 9. The leaving flow, the entering flow, the net flow and outranking index of each candidate.

\begin{tabular}{lcccc}
\hline & $\boldsymbol{\varphi}^{+}\left(\boldsymbol{A}_{\boldsymbol{i}}\right)$ & $\boldsymbol{\varphi}^{-}\left(\boldsymbol{A}_{\boldsymbol{i}}\right)$ & $\boldsymbol{\varphi}\left(\boldsymbol{A}_{\boldsymbol{i}}\right)$ & $\boldsymbol{O T I}\left(\boldsymbol{A}_{\boldsymbol{i}}\right)$ \\
\hline$A_{2}$ & 3.5602 & 2.0710 & 1.4893 & 0.5827 \\
$A_{4}$ & 4.2924 & 1.6354 & 2.6570 & 0.6476 \\
$A_{6}$ & 1.9195 & 3.9636 & -2.0440 & 0.3864 \\
$A_{7}$ & 3.0959 & 3.3062 & -0.2102 & 0.4883 \\
$A_{11}$ & 0.8021 & 6.3876 & -5.5855 & 0.1897 \\
$A_{12}$ & 4.3260 & 1.3237 & 3.0023 & 0.6668 \\
$A_{13}$ & 4.1420 & 2.0393 & 2.1027 & 0.6168 \\
$A_{14}$ & 2.0424 & 4.1897 & -2.1473 & 0.3807 \\
$A_{18}$ & 3.6810 & 1.6495 & 2.0314 & 0.6129 \\
$A_{19}$ & 2.6544 & 3.9501 & -1.2957 & 0.4280 \\
\hline
\end{tabular}

Step 8. Based on the support ratios of quantitative and qualitative criteria, a comprehensive support index of each qualified applicant can be calculated as Table 10. If the preference index of the final decision-maker is 0.5 (i.e., $D_{P}=0.5$ ), then the ranking order of all qualified applicants in accordance with comprehensive support indices is $\left\{A_{12}>A_{4}>A_{2}>A_{18}>A_{14}>A_{13}>A_{6}>A_{19}>A_{7}>A_{11}\right\}$.

Table 10. Comprehensive support indices of candidates.

\begin{tabular}{cccc}
\hline & $S R_{\text {quan }}\left(A_{\boldsymbol{i}}\right)$ & $\boldsymbol{S R}_{\text {qual }}\left(\boldsymbol{A}_{\boldsymbol{i}}\right)$ & $\boldsymbol{S R}_{\boldsymbol{c o m p}}\left(\boldsymbol{A}_{\boldsymbol{i}}\right)$ \\
\hline$A_{2}$ & 0.1092 & 0.1165 & 0.1129 \\
$A_{4}$ & 0.0971 & 0.1295 & 0.1133 \\
$A_{6}$ & 0.1156 & 0.0773 & 0.0965 \\
$A_{7}$ & 0.0791 & 0.0977 & 0.0884 \\
$A_{11}$ & 0.0840 & 0.0379 & 0.0610 \\
$A_{12}$ & 0.1019 & 0.1334 & 0.1176 \\
$A_{13}$ & 0.0829 & 0.1234 & 0.1031 \\
$A_{14}$ & 0.1352 & 0.0761 & 0.1057 \\
$A_{18}$ & 0.1028 & 0.1226 & 0.1127 \\
$A_{19}$ & 0.0922 & 0.0856 & 0.0889 \\
\hline
\end{tabular}

If the preference index of the final decision-maker changes, then the comprehensive support index of each qualified applicant will be different as Figure 7. In addition, the ranking order of qualified applicants will change in accordance with $D_{P}$ as Figure 8 . As we know, the final decision-maker can select the suitable applicants flexibly with a different preference index $\left(D_{P}\right)$.

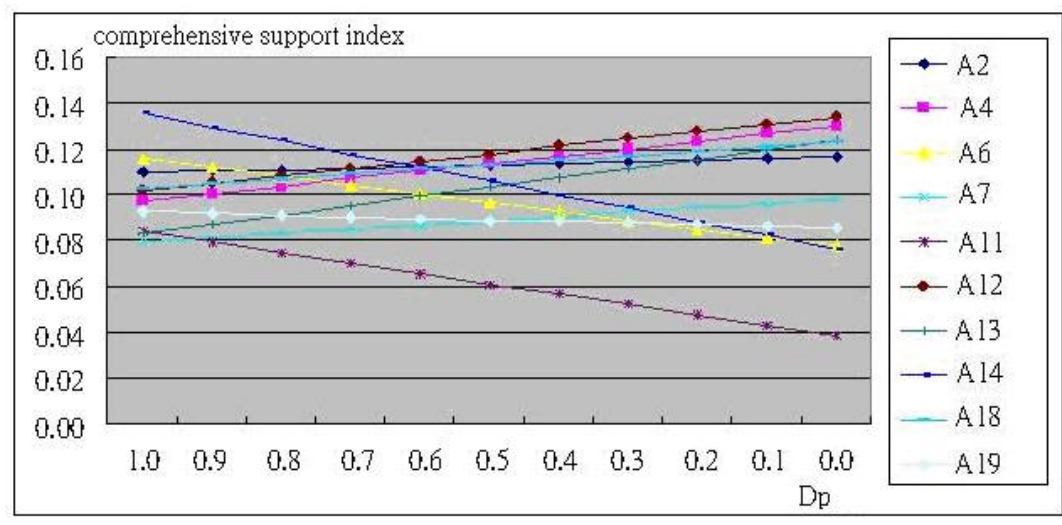

Figure 7. Comprehensive support indices of candidates with different $D_{P}$. 


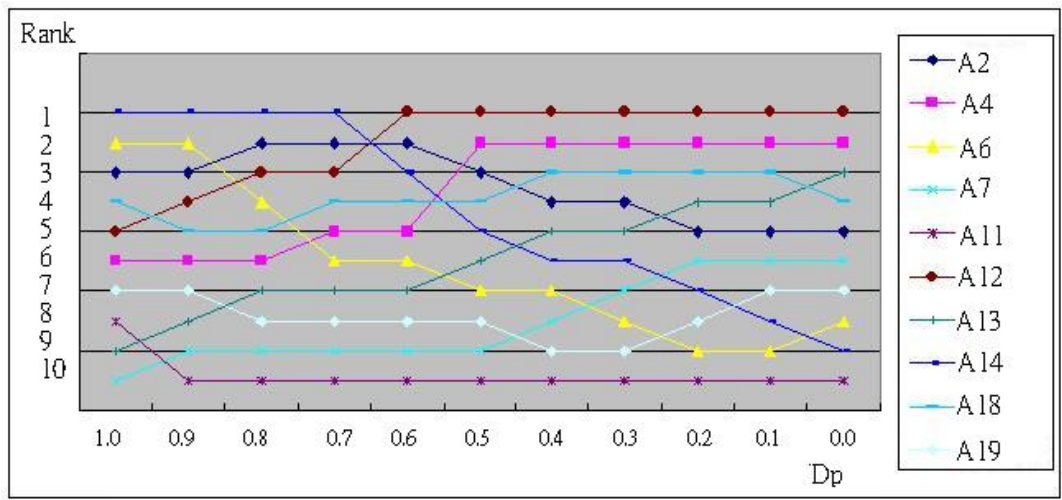

Figure 8. The ranking order of candidates with different $D_{P}$.

\section{Simulation and Effectiveness Explanation}

In order to illustrate the effectiveness of the TPMCDM method for dealing with the personnel selection problem, we implement data simulation to compare TPMCDM with TOPSIS [6-8] and PROMETHEE [51] methods with linguistic variables. The linguistic TOPSIS (L-TOPSIS) and linguistic PROMETHEE (L-PROMETHEE) methods are one phase for determining the ranking order of all applicants in accordance with quantitative and qualitative criteria simultaneously. Based on the proposed example, we generate new data of each applicant by using random variables. The range of generating new data with respect to criteria are shown in Table 11, and 100,000 simulations are executed to make the comparisons between TPMCDM and the others.

Table 11. The data range of each criterion.

\begin{tabular}{cccc}
\hline Criterion & Description & Data Type & Range \\
\hline$C_{1}$ & English ability & Quantitative Data & $10 \sim 990$ \\
$C_{2}$ & Work experience & Quantitative Data & $1 \sim 10$ \\
$C_{3}$ & Educational Background & Quantitative Data & $1,2,3,4$ \\
$C_{4}$ & License & Quantitative Data & $1 \sim 10$ \\
$C_{5}$ & Communication skill & Qualitative Data & Expert $\mathrm{E}_{1}\left(s_{0}^{5}, 0\right) \sim\left(s_{4}^{5}, 0\right)$ \\
$C_{6}$ & Innovation ability & Qualitative Data & Expert $\mathrm{E}_{2}\left(s_{0}^{7}, 0\right) \sim\left(s_{6}^{7}, 0\right)$ \\
$C_{7}$ & Advertise design ability & Qualitative Data & Expert $\mathrm{E}_{3}\left(s_{0}^{9}, 0\right) \sim\left(s_{8^{\prime}}^{9}, 0\right)$ \\
$C_{8}$ & Emotional steadiness & Qualitative Data & \\
\hline
\end{tabular}

If the better applicants are selected by the TPMCDM method, which overlaps with other methods, then the two-phase method is effective for reducing the cost of interview time. In order to compute the overlapped ratios between the TPMCDM method and the other methods, the top ten applicants are selected from all applicants by the TPMCDM, L-TOPSIS, and L-PROMETHEE methods, respectively. Therefore, we define the coverage rate $C V_{\text {ltop }}$ to express the overlap degree of the top ten applicants between the TPMCDM method and L-TOPSIS. We then define the coverage rate $C V_{\text {lpro }}$ to express the degree of overlap of the top ten applicants between the TPMCDM method and L-PROMETHEE. We compute $C V_{\text {ltop }}$ and $C V_{\text {lpro }}$ as:

$$
\begin{aligned}
& C V_{\text {ltop }}=\operatorname{Count}\left\{A_{i} \mid \operatorname{Rank}_{\text {ltop }}\left(A_{i}\right) \leq 10 \& \operatorname{Rank}_{\text {tpm }}\left(A_{i}\right) \leq 10\right\} / 10 \\
& C V_{\text {lpro }}=\operatorname{Count}\left\{A_{i} \mid \operatorname{Rank}_{\text {lpro }}\left(A_{i}\right) \leq 10 \& \operatorname{Rank}_{\text {tpm }}\left(A_{i}\right) \leq 10\right\} / 10,
\end{aligned}
$$

where $\operatorname{Rank}_{\text {ltop }}\left(A_{i}\right)$ is the rank order of applicant $A_{i}$ by the L-TOPSIS method. Rank lpro $\left(A_{i}\right)$ is the rank order of applicant $A_{i}$ by the L-PROMETHEE method. $\operatorname{Rank}_{t p m}\left(A_{i}\right)$ is the rank order of applicant $A_{i}$ by the TPMCDM method. 
As we know, the TPMCDM method eliminates some applicants in the first step for saving on interview time and cost. Under this situation, the elimination ratio and coverage rate exhibit a trade-off relationship to select the better applicants for managers. A higher elimination ratio at the first step may produce a lower coverage rate. A lower elimination ratio at the first step cannot reduce the interview time and cost for managers effectively.

According to the results of Table 12 and Figure 9, we find that larger elimination ratios truly produce smaller coverage rates. For example, if managers want to save $20 \%$ of interview time (elimination rate is $20 \%$ ) to select applicants, then the coverage ratios are $84.17 \%$ and $80.64 \%$ between the TPMCDM method and the L-TOPSIS and L-PROMETHEE methods, respectively. However, if managers want to save $10 \%$ of interview time (elimination rate is $10 \%$ ) to select the applicants, then the coverage ratios are $92.81 \%$ and $91.16 \%$ between the TPMCDM method and L-TOPSIS and L-PROMETHEE methods, respectively. Thus, the elimination ratios and coverage rates have a trade-off relationship. A decision-maker should consider and decide the relative importance between experts' time and personnel selection decision quality.

Table 12. The coverage rates with different elimination rates.

\begin{tabular}{cccccc}
\hline \multirow{2}{*}{$\begin{array}{c}\text { Coverage } \\
\text { Rate }\end{array}$} & \multicolumn{5}{c}{ Elimination Rate } \\
\cline { 2 - 6 } & $\mathbf{5 \%}$ & $\mathbf{1 0 \%}$ & $\mathbf{1 5 \%}$ & $\mathbf{2 0 \%}$ & $\mathbf{2 5 \%}$ \\
\hline$C V_{\text {top }}$ & $96.64 \%$ & $92.81 \%$ & $88.64 \%$ & $84.17 \%$ & $79.48 \%$ \\
$C V_{\text {pro }}$ & $95.92 \%$ & $91.16 \%$ & $86.02 \%$ & $80.64 \%$ & $75.15 \%$ \\
\hline Coverage & \multicolumn{5}{c}{ Elimination Rate } \\
Rate & $\mathbf{3 0 \%}$ & $\mathbf{3 5 \%}$ & $\mathbf{4 0 \%}$ & $\mathbf{4 5 \%}$ & $\mathbf{5 0 \%}$ \\
$C V_{\text {top }}$ & $74.64 \%$ & $69.66 \%$ & $64.55 \%$ & $59.35 \%$ & $54.04 \%$ \\
$C V_{\text {pro }}$ & $69.57 \%$ & $64.00 \%$ & $58.42 \%$ & $52.87 \%$ & $47.37 \%$ \\
\hline
\end{tabular}

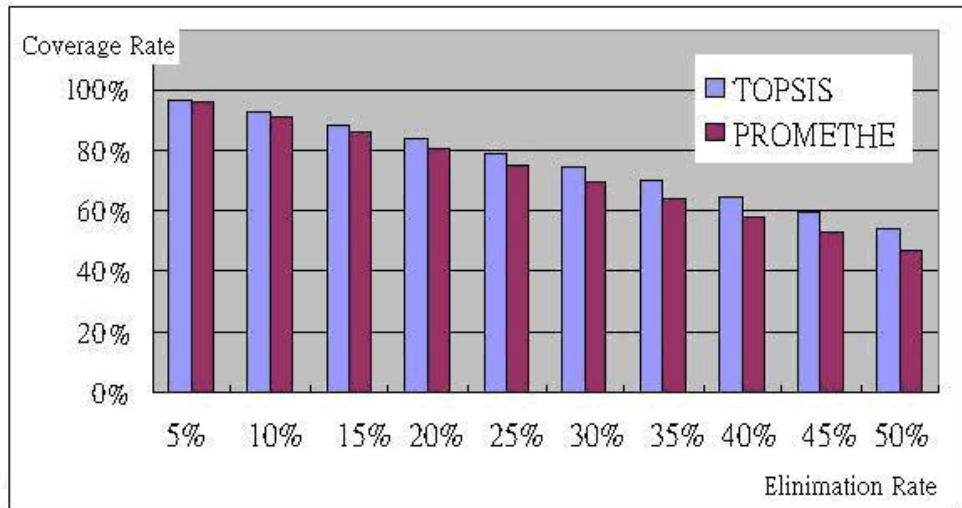

Figure 9. The relationship of coverage and elimination rates.

The average rank order of each selected applicant by the TPMCDM method is computed to compare with the linguistic TOPSIS and linguistic PROMETHEE methods, respectively. According to experiment result (shown as Figure 10), we know that a lower rank order of the applicant in the TPMCDM method acquires a lower average rank order in the L-TOPSIS and L-PROMETHEE methods, respectively. The rank order distribution of each selected applicant by the TPMCDM method is also computed in the linguistic TOPSIS method as Figure 11. The rank order distribution of each selected applicant by the TPMCDM method is also computed in the linguistic PROMETHEE method as Figure 12. Based on the above analysis results, we find that the TPMCDM method can help managers to select suitable employees and can effectively reduce the interview time and cost during the process of personnel recruitment. 


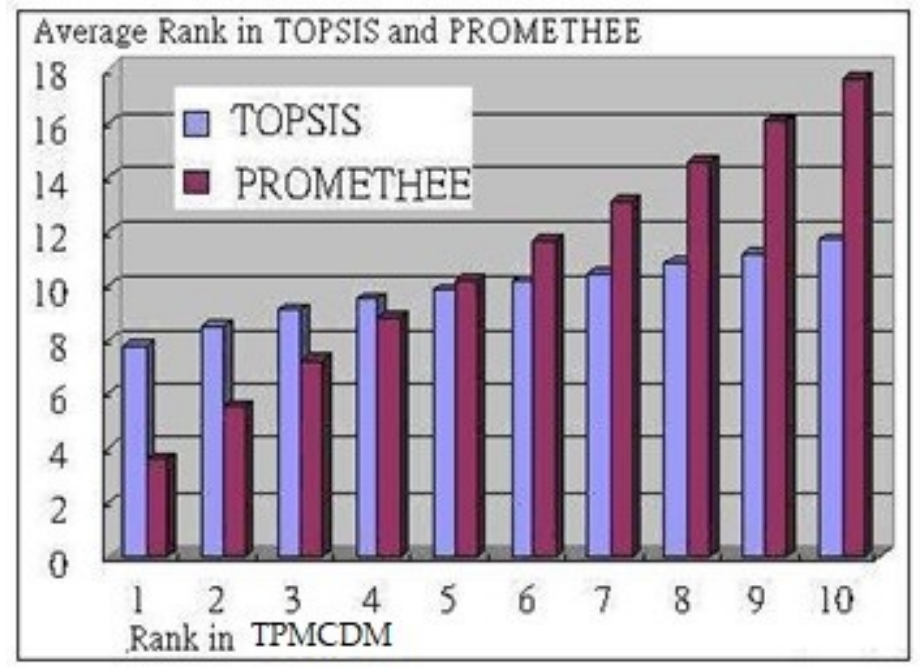

Figure 10. The average rank of selected applicants by two-phase multi-criteria decision-making (TPMCDM) are computed in linguistic TOPSIS and preference ranking organization method for enrichment evaluation (PROMETHEE).

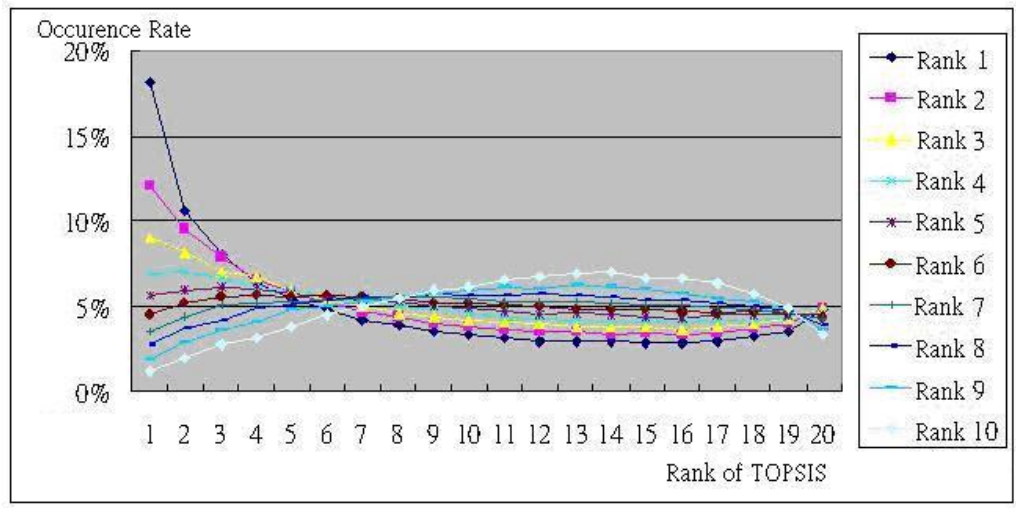

Figure 11. The rank distribution of selected applicants by TPMCDM are computed in linguistic TOPSIS.

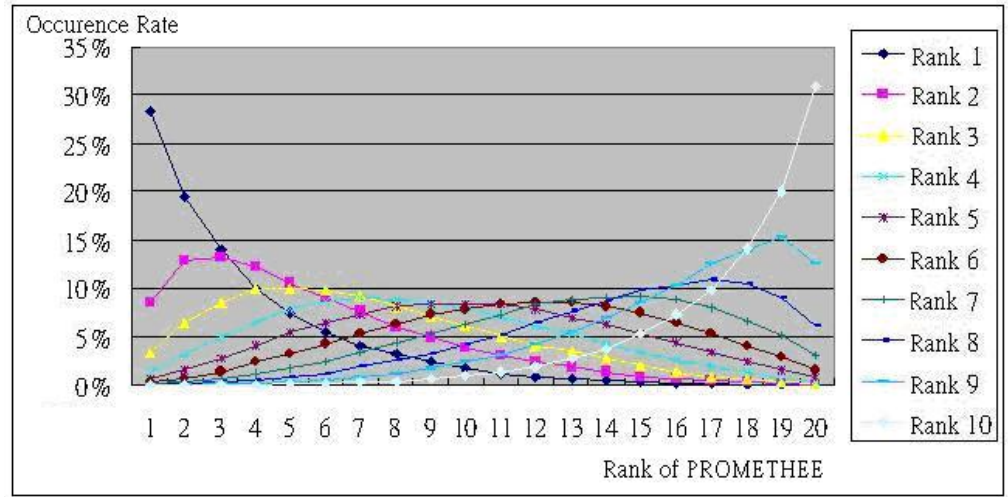

Figure 12. The rank distribution of selected applicants by TPMCDM are computed in linguistic PROMETHEE.

\section{Conclusions}

When facing a global competitive environment, enterprises know that the selection of suitable persons is key to their survival. However, it is costly to review and evaluate huge numbers of applicants. In order to cope with the personnel selection problem efficiently and effectively, a two-phase multiple-criteria decision-making (TPMCDM) method is presented herein to select suitable 
persons for the right positions by considering quantitative and qualitative factors at the same time. The contributions and advantages of the systematic method proposed in this paper can be summarized as follows.

(1) According to the two-phase MCDM method, some unsuitable applicants will be filtered effectively by considering quantitative information at the first stage. In other words, the two-phase MCDM method can reduce the time for reviewing and evaluating the huge numbers of applicants at the same time.

(2) To avoid subjective judgment by experts to determine the weights of all criteria, the entropy method automatically computes according to the data distribution.

(3) By combining the support ratios of qualitative with quantitative information, a comprehensive support index is defined to determine the ranking order of all candidates effectively. Considering the different importance of qualitative and quantitative factors, managers can adjust their preference to compute the comprehensive support index and select the suitable employee.

(4) In this research, the elimination ratios and coverage rates have a trade-off relationship. Enterprises can use the proposed method by justifying that the elimination ratios save decision makers' time according to the enterprises' actual demand.

The proposed model can reduce the overload of reviewing applicants for managers. The TPMCDM method of personnel recruitment provides an objective and systematic process to handle the personnel selection problem with huge numbers of applicants. The multi-type linguistic variables based on 2-type linguistic terms are used to express the evaluation values of decision-makers in this paper. However, it is not easy to define the suitable type linguistic variables for experts to express their subjective opinions. It is the limitation of the proposed method to deal with the personnel selection problem based on huge amounts of applicants. To enhance the practical value of the two-phase multiple-criteria decision-making (TPMCDM) method, one can design and develop a decision support system based on the proposed model in the near future.

Author Contributions: Conceptualization, C.-T.C.; methodology, C.-T.C. and W.-Z.H.; software, W.-Z.H.; validation, C.-T.C. and W.-Z.H.; formal analysis, C.-T.C. and W.-Z.H.; data curation, W.-Z.H.; writing-original draft preparation, C.-T.C.; writing-review and editing, C.-T.C.; visualization, W.-Z.H.; project administration, C.-T.C. All authors have read and agreed to the published version of the manuscript.

Funding: This research received no external funding.

Acknowledgments: This work was supported partially by Ministry of Science and Technology, Taiwan. The project No. is "MOST 108-2410-H-239-010-MY2".

Conflicts of Interest: The authors declare no conflict of interest.

\section{References}

1. Chien, C.F.; Chen, L.F. Data mining to improve personnel selection and enhance human capital: A case study in high-technology industry. Expert Syst. Appl. 2008, 34, 280-290. [CrossRef]

2. Caligiuri, P.; Tarique, I.; Jacobs, R. Selection for international assignments. Hum. Resour. Manag. Rev. 2009, 19, 251-262. [CrossRef]

3. Figueira, J.; Greco, S.; Ehrgott, M. Multiple Criteria Decision Analysis: State of the Art Surveys; Springer: Berlin/Heidelberg, Germany, 2005.

4. Liu, H.C.; Chen, X.Q.; Duan, C.Y.; Wang, Y.M. Failure mode and effect analysis using multi-criteria decision making methods: A systematic literature review. Comput. Ind. Eng. 2019, 135, 881-897. [CrossRef]

5. Wanga, X.; Triantaphylloub, E. Ranking irregularities when evaluating alternatives by using some ELECTRE methods. Omega 2008, 36, 45-63. [CrossRef]

6. Hwang, C.; Yoon, K. Multiple Attribute Decision Making: Methods and Application; Springer: Berlin/Heidelberg, Germany, 1981.

7. Kelemenis, A.; Askounis, D. A new TOPSIS-based multi-criteria approach to personnel selection. Expert Syst. Appl. 2010, 37, 4999-5008. [CrossRef] 
8. Korkmaz, O. Personnel Selection Method Based on TOPSIS Multi-Criteria Decision-Making Method. Int. J. Econ. Adm. Stud. 2019, in press. [CrossRef]

9. Amiri, M.P. Project selection for oil-fields development by using the AHP and fuzzy TOPSIS methods. Expert Syst. Appl. 2010, 37, 6218-6224. [CrossRef]

10. Dağdeviren, M.; Yavuz, S.; Kılınç, N. Weapon selection using the AHP and TOPSIS methods under fuzzy environment. Expert Syst. Appl. 2009, 36, 8143-8151. [CrossRef]

11. Wang, Y.M.; Taha, M.S.E. Fuzzy TOPSIS method based on alpha level sets with an application to bridge risk assessment. Expert Syst. Appl. 2006, 31, 309-319. [CrossRef]

12. Saremi, M.; Mousavi, F.; Sanayei, A. TQM consultant selection in SMEs with TOPSIS under fuzzy environment. Expert Syst. Appl. 2009, 36, 2742-2749. [CrossRef]

13. Chen, C.T.; Hung, W.Z. A new decision-making method for stock portfolio selection based on computing with linguistic assessment. Adv. Decis. Sci. 2009, 2009, 897024. [CrossRef]

14. Brans, J.P.; Vincke, P.; Mareschal, B. How to select and how to rank projects: The PROMETHEE method. Eur. J. Oper. Res. 1986, 24, 228-238. [CrossRef]

15. Goumas, M.; Lygerou, V. An extension of the PROMETHEE method for decision making in fuzzy environment: Ranking of alternative energy exploitation projects. Eur. J. Oper. Res. 2000, 123, 606-613. [CrossRef]

16. Halouani, N.; Chabchoub, H.; Martel, J.M. PROMETHEE-MD-2T method for project selection. Eur. J. Oper. Res. 2009, 195, 841-849. [CrossRef]

17. Albadvi, A.; Chaharsooghi, K.; Esfahanipour, A. Decision making in stock trading: An application of PROMETHEE. Eur. J. Oper. Res. 2007, 177, 673-683. [CrossRef]

18. Mousavi, M.M.; Lin, J. The application of PROMETHEE Multi-criteria Decision Aid in Financial Decision Making: Case of Distress Prediction Models Evaluation. Expert Syst. Appl. 2020, 159, 113438, in press. [CrossRef]

19. Pavi, I.; Babi, Z. The use of the PROMETHEE method in the location choice of a production system. Int. J. Prod. Econ. 1991, 23, 165-174. [CrossRef]

20. Shannon, C.E. The mathematical theory of communications. Bell Syst. Tech. J. 1947, 27, 379-423. [CrossRef]

21. Tian, Q.H.; Du, Y.X. Study of performance evaluation for mechanical products based on entropy fuzzy comprehensive review. J. China Man Inform. 2004, 33, 97-99.

22. Zou, Z.H.; Yun, Y.; Sun, J.N. Entropy method for determination of weight of evaluating indicators in fuzzy synthetic evaluation for water quality assessment. J. Environ. Sci. 2006, 18, 1020-1023. [CrossRef]

23. Guo, C.Z. Study on the evaluating method of entropy coefficient for stock investment value. J. Nankai Econ. Stud. 2001, 5, 65-67.

24. Marchettini, N.; Pulselli, R.M.; Rossi, F.; Tiezzi, E. Entropy. In Encyclopedia of Ecology; Elsevier Science: Amsterdam, The Netherlands, 2008; pp. 1297-1305.

25. Herrera, F.; Martinez, L. A 2-tuple fuzzy linguistic representation model for computing with words. IEEE Trans. Fuzzy Syst. 2000, 8, 746-752.

26. Xu, Z.S. Deviation measures of linguistic preference relations in group decision making. Omega 2005, 33, 249-254. [CrossRef]

27. Herrera-Viedma, E.; Cordón, O.; Luque, M.; Lopez, A.G.; Muñoz, A.M. A model of fuzzy linguistic IRS based on multigranular linguistic information. Int. J. Approx. Reason. 2003, 34, 221-239. [CrossRef]

28. Ertugrul Karsak, E. A fuzzy multiple objective programming approach for personnel selection. In Proceedings of the 2000 IEEE International Conference on Systems, Man and Cybernetics, Nashville, TN, USA, 8-11 October 2000; Volume 3, pp. 2007-2012.

29. Korvin, A.D.; Shipley, M.F.; Kleyle, R. Utilizing fuzzy compatibility of skill sets for team selection in multi-phase projects. J. Eng. Technol. Manag. 2002, 19, 307-319. [CrossRef]

30. Chien, C.F.; Chen, L.F. Using rough set theory to recruit and retain high-potential talents for semiconductor. IEEE Trans. Semicond. Manuf. 2007, 20, 528-541. [CrossRef]

31. Gungor, Z.; Serhadloglu, G.; Kesen, S.E. A fuzzy AHP approach to personnel selection problem. Appl. Soft Comput. 2009, 9, 641-646. [CrossRef]

32. Celik, M.; Kandakoglu, A.; Deha Er, I. Structuring fuzzy integrated multi-stages evaluation model on academic personnel recruitment in MET institutions. Expert Syst. Appl. 2009, 36, 6918-6927. [CrossRef]

33. Fan, Z.P.; Feng, B.; Jiang, Z.Z.; Fu, N. A method for member selection of R\&D teams using the individual and collaborative information. Expert Syst. Appl. 2009, 36, 8313-8323. 
34. Feng, B.; Jiang, Z.Z.; Fan, Z.P.; Fu, N. A method for member selection of cross-functional teams using the individual and collaborative performances. Eur. J. Oper. Res. 2010, 203, 652-661. [CrossRef]

35. Zhang, S.F.; Liu, S.Y. A GRA-based intuitionistic fuzzy multi-criteria group decision making method for personnel selection. Expert Syst. Appl. 2011, 38, 11401-11405. [CrossRef]

36. Chen, C.T.; Pai, P.F.; Hung, W.Z. Applying Linguistic VIKOR and Knowledge Map in Personnel Selection. Asia Pac. Manag. Rev. 2011, 16, 491-502.

37. Bangerter, A.; Roulin, N.; König, C.J. Personnel selection as a signaling game. J. Appl. Psychol. 2012, 97, 719-738. [CrossRef]

38. Afshari, A.R.; Yusuff, R.M.; Derayatifar, A.R. Linguistic extension of fuzzy integral for group personnel selection problem. Arab. J. Sci. Eng. 2013, 38, 2901-2910. [CrossRef]

39. Sang, X.; Liu, X.; Qin, J. An analytical solution to fuzzy TOPSIS and its application in personnel selection for knowledge-intensive enterprise. Appl. Soft Comput. 2015, 30, 190-204. [CrossRef]

40. Ji, P.; Zhang, H.Y.; Wang, J.Q. A projection-based TODIM method under multi-valued neutrosophic environments and its application in personnel selection. Neural Comput. Appl. 2018, 29, 221-234. [CrossRef]

41. Heidary Dahooie, J.; Beheshti Jazan Abadi, E.; Vanaki, A.S.; Firoozfar, H.R. Competency-based IT personnel selection using a hybrid SWARA and ARAS-G methodology. Hum. Factors Ergon. Manuf. Serv. Ind. 2018, 28, 5-16. [CrossRef]

42. Yalçın, N.; Yapıcı Pehlivan, N. Application of the fuzzy CODAS method based on fuzzy envelopes for hesitant fuzzy linguistic term sets: A case study on a personnel selection problem. Symmetry 2019, 11, 493. [CrossRef]

43. Chuang, Y.C.; Hu, S.K.; Liou, J.J.; Tzeng, G.H. A data-driven MADM model for personnel selection and improvement. Technol. Econ. Dev. Econ. 2020, 26, 751-784. [CrossRef]

44. Kilic, H.S.; Demirci, A.E.; Delen, D. An integrated decision analysis methodology based on IF-DEMATEL and IF-ELECTRE for personnel selection. Decis. Support Syst. 2020, 137, 113360, in press. [CrossRef]

45. Krishankumar, R.; Premaladha, J.; Ravichandran, K.S.; Sekar, K.R.; Manikandan, R.; Gao, X.Z. A novel extension to VIKOR method under intuitionistic fuzzy context for solving personnel selection problem. Soft Comput. 2020, 24, 1063-1081. [CrossRef]

46. Ozdemir, Y.; Nalbant, K.G. Personnel selection for promotion using an integrated consistent fuzzy preference relation-Fuzzy analytic hierarchy process methodology: A real case study. Asian. J. Interdiscip. Res. 2020, 3, 219-236. [CrossRef]

47. Herrera, F.; Martinez, L. A model based on linguistic 2-tuples for dealing with multigranular hierarchical linguistic contexts in multi-expert decision-making. IEEE Trans. Syst. Man Cybern. Part B 2001, 31, 227-234. [CrossRef] [PubMed]

48. Tai, W.S.; Chen, C.T. A new evaluation model for intellectual capital based on computing with linguistic variable. Expert Syst. Appl. 2009, 36, 3483-3488. [CrossRef]

49. Herrera-Viedma, E.; Herrera, F.L.; Martínez, L.; Herrera, J.C.; López, A.G. Incorporating filtering techniques in a fuzzy linguistic multi-agent model for information gathering on the web. Fuzzy Sets Syst. 2004, 148, 61-83. [CrossRef]

50. Herrera, F.; Martinez, L.; Sanchez, P.J. Managing non-homogeneous information in group decision making. Eur. J. Oper. Res. 2005, 166, 115-132. [CrossRef]

51. Chen, C.T.; Pai, P.F.; Hung, W.Z. An integrated methodology using linguistic PROMETHEE and maximum deviation method for third-party logistics supplier selection. Int. J. Comput. Intell. Sys. 2010, 3, 438-451. [CrossRef]

(C) 2020 by the authors. Licensee MDPI, Basel, Switzerland. This article is an open access article distributed under the terms and conditions of the Creative Commons Attribution (CC BY) license (http://creativecommons.org/licenses/by/4.0/). 